\title{
Computing branchwidth via efficient triangulations and blocks
}

\author{
Fedor Fomin* $\quad$ Frédéric Mazoit ${ }^{\dagger} \quad$ Ioan Todinca ${ }^{\ddagger}$
}

\begin{abstract}
Minimal triangulations and potential maximal cliques are the main ingredients for a number of polynomial time algorithms on different graph classes computing the treewidth of a graph. Potential maximal cliques are also the main engine of the fastest so far $\mathcal{O}\left(1.9601^{n}\right)$ time exact treewidth algorithm. Based on the recent results of Mazoit, we define the structures that can be regarded as minimal triangulations and potential maximal cliques for branchwidth: efficient triangulations and blocks. We show how blocks can be used to construct an algorithm computing the branchwidth of a graph on $n$ vertices in time $(2+\sqrt{3})^{n} \cdot n^{O(1)}$.
\end{abstract}

\section{Introduction}

Treewidth is one of the most basic parameters in graph algorithms and it plays an important role in structural graph theory. Treewidth serves as the main tools in Robertson and Seymour's Graph Minors project [18]. It is well known that many intractable problems can be solved in polynomial (and very often in linear time) when the input is restricted to graphs of bounded treewidth. See [3] for a comprehensive survey.

The branchwidth is strongly related to treewidth. It is known that for any graph $G$, bw $(G) \leq$ $\operatorname{tw}(G)+1 \leq 1.5 \cdot \operatorname{bw}(G)$. Both bounds are tight and achievable on trees and complete graphs. Branchwidth was introduced by Robertson \& Seymour and it appeared to be even more appropriate tool than treewidth for Graph Minor Theory. Since both parameters are so close, one can expect that the algorithmic behavior of the problems is also quite similar. However, this is not true. For example, on planar graphs branchwidth is solvable in polynomial time [21] while computing the treewidth of a planar graph in polynomial time is a long standing open problem. Even more striking example was observed by Kloks et al. in [14]: it appeared that computing branchwidth is NP hard even on split graphs. Note that the treewidth of a split graph can be found in linear time.

The last decade has led to much research in fast exponential-time algorithms. Examples of recently developed exponential algorithms are algorithms for Maximum Independent Set [13, 19], (Maximum) Satisfiability [7, 12, 17, 20, 23], Coloring [2, 5, 8], and many others (see the recent survey written by Woeginger [24] for an overview). There are several relatively simple algorithms based on dynamic programming computing the treewidth of a graph on $n$ vertices in time $2^{n}$.

\footnotetext{
${ }^{*}$ Department of Informatics, University of Bergen, N-5020 Bergen, Norway, fomin@ii . uib. no

${ }^{\dagger}$ LIF, Université de provence 13453 Marseille Cedex 13 France, Frederic. Ma zoitelif.univ-mrs. fr

${ }^{\ddagger}$ LIFO, Université d’Orléans 45067 Orléans Cedex 2 France, Ioan. Todinca@ li fo . univ-or leans . fr
} 
$n^{O(1)}$ which with more careful analyze can be speed-up to $\mathcal{O}\left(1.9601^{n}\right)$ [9]. No such algorithm is known for branchwidth. The only nontrivial algorithm for branchwidth we were aware can be obtained by using deep min-max theorems of Robertson \& Seymour [18] relating branchwidth and tangles. Then by playing with tangle axioms one can reduce the search space and perform dynamic programming to construct optimal tangles in time $4^{n} \cdot n^{O(1)}$. (We leave the details in this extended abstract.)

Thus treewidth seems to be more simple problem for design of exponential time algorithms than branchwidth. The explanation to that can be that all known exact algorithms for treewidth exploit the relations between treewidth, minimal triangulations, minimal separators and potential maximal cliques. Mazoit in [15] observed that the branchwidth also can be seen as a triangulation problem. However, while for treewidth one can work only with minimal triangulations the situation with branchwidth is more complicated. Lucky enough we still can use some specific triangulations, which we call efficient triangulations. In this paper we adopt the techniques of Mazoit to discover the analogue of potential maximal cliques for branchwidth, we call these structures by blocks. Potential maximal cliques are extremely useful tools in work with treewidth $[4,9]$. We believe that blocks can also be useful to work with branchwidth. To exemplify that we show how blocks can be used to compute branchwidth in time $(2+\sqrt{3})^{n} \cdot n^{O(1)}$. Note that this is the fastest known exact algorithm for this problem.

\section{Basic definitions}

We denote by $G=(V, E)$ a finite undirected and simple graph with $|V|=n$ vertices and $|E|=m$ edges. Throughout this paper we use a modified big-Oh notation that suppresses all polynomially bounded factors. For functions $f$ and $g$ we write $f(n)=\mathcal{O}^{*}(g(n))$ if $f(n)=g(n) \cdot n^{\mathcal{O}(1)}$.

For any non-empty subset $W \subseteq V$, the subgraph of $G$ induced by $W$ is denoted by $G[W]$. If $S$ is a set of vertices, we denote by $G-S$ the graph $G[V \backslash S]$. The neighborhood of a vertex $v$ is $N(v)=\{u \in V:\{u, v\} \in E\}$ and for a vertex set $S \subseteq V$ we put $N(S)=\bigcup_{v \in S} N(v) \backslash S$. A clique $C$ of a graph $G$ is a subset of $V$ such that all the vertices of $C$ are pairwise adjacent. Let $\omega(G)$ denote the maximum clique size of $G$.

A graph $G$ is chordal if every cycle of $G$ with at least four vertices has a chord, that is an edge between two non-consecutive vertices of the cycle. Consider an arbitrary graph $G=(V, E)$, and a supergraph $H=(V, F)$ of $G$ (i.e. $E \subseteq F)$. We say that $H$ is a triangulation of $G$ if $H$ is chordal. Moreover, if no strict sub-graph of $H$ is a triangulation of $G$, then $H$ is called a minimal triangulation.

The notion of branchwidth is due to Robertson and Seymour [18]. A branch decomposition of a graph $G=(V, E)$ is a pair $(T, \tau)$ in which $T=\left(V_{T}, E_{T}\right)$ is a ternary tree (i.e. each node is of degree one or three) and $\tau$ is a function mapping each edge of $G$ on a leaf of $T$. The vertices of $T$ will be called nodes and its edges will be called branches. For any branch $e \in E_{T}$, let $T_{1}(e)$ and $T_{2}(e)$ be the subtrees obtained from $T$ by removing $e$. Let lab $(e)$ be the set of vertices of $G$ both incident to edges mapped on $T_{1}(e)$ and $T_{2}(e)$. The maximum of $\left\{|l a b(e)|, e \in E_{T}\right\}$, is called the width of the branch decomposition. The branchwidth of a graph $G(\mathrm{bw}(G))$ is the minimum width over all branch decompositions of $G$. Note that the definitions of branch decomposition and branch-width also apply to hypergraphs. As pointed by Robertson and Seymour, the definition 
of branch decomposition can be relaxed. A relaxed branch decomposition of $G=(V, E)$ is a couple $(T, \tau)$ where $T$ is an arbitrary tree and $\tau$ is an application mapping each edge of $G$ to at least one leaf of $T$. The labels of the branches and the width of the decomposition are defined as before. From any relaxed branch decomposition we can construct a branch decomposition without increasing the width.

The branchwidth is strongly related to a well-known graph parameter introduced by Robertson and Seymour, namely the treewidth. One of the equivalent definitions for treewidth is $\operatorname{tw}(G)=$ $\min \{\omega(H)-1 \mid H$ is a triangulation of $G\}$. Robertson and Seymour show that the two parameters differ by at most a factor of 1.5. More precisely, for any graph $G$ we have $\operatorname{bw}(G) \leq$ $\operatorname{tw}(G)+1 \leq 1.5 \mathrm{bw}(G)$. In particular, if $G$ is a complete graph, its treewidth is $n-1$, while its branchwidth is $\lceil 2 n / 3\rceil$ (see [18]). Clearly, when computing the treewidth of a graph we can restrict to minimal triangulations. This observation and the study of minimal triangulations of graphs led to several results about treewidth computation, including an exact algorithm in $\mathcal{O}^{*}\left(1.961^{n}\right)$ time.

The branch decompositions of a graph can also be associated to triangulations. Indeed, given a branch decomposition $(T, \tau)$ of $G=(V, E)$, we can associate to each $x \in V$ the subtree of $T$ covering all the leaves of $T$ containing edges incident to $x$. It is well-known that the intersection graph of the sub-trees of a tree is chordal [10]. Thus the intersection graph of the trees $T_{x}$ is a triangulation $H(T, \tau)$ of $G$. Note that for each branch $e \in E_{T}, \operatorname{lab}(e)$ is the set of vertices $x$ such that $e$ belongs to $T_{x}$. In particular, $\operatorname{lab}(e)$ induces a clique in $H(T, \tau)$, not necessarily maximal. (We shall point out later that, for each maximal clique $\Omega$ of $H(T, \tau)$, there exists a node $u$ of $T$ such that $u \in T_{x}$ for all $x \in \Omega$.)

The first big difference with treewidth is that there exist examples of graphs for which any optimal branch decomposition leads to non-minimal triangulations [15]. Therefore the many existing tools about minimal triangulations are not sufficient in our case. The second important difference is that the branchwidth problem remains NP-hard even for a restricted class of chordal graphs, the split graphs [14]. Nevertheless, our technique for computing the branchwidth relies on a structural result stating that, for any graph $G$, there is an optimal branch decomposition $(T, \tau)$ such that $H(T, \tau)$ is an efficient triangulation of $G$. The efficient decomposition, defined in the next section, behave somehow similarily to minimal decompositions. In order to obtain our exact algorithm for branchwidth, we will combine this observation with an exponential algorithm computing the branchwidth of hyper-cliques.

\section{Branchwidth and efficient triangulations}

Let $a$ and $b$ be two non adjacent vertices of a graph $G=(V, E)$. A set of vertices $S \subseteq V$ is an $a, b$-separator if in the graph $G-S a$ and $b$ in are in different connected components. $S$ is a minimal $a, b$-separator if no proper subset of $S$ is an $a, b$-separator. We say that $S$ is a minimal separator of $G$ if there are two vertices $a$ and $b$ such that $S$ is a minimal $a, b$-separator. We denote by $\mathcal{C}(S)$ the set of connected components of $G-S$ and by $\Delta_{G}$ the set of all minimal separators of $G$.

Definition 1. A triangulation $H$ of $G$ is efficient if

1. each minimal separator of $H$ is also a minimal separator of $G$; 
2. for each minimal separator $S$ of $H$, the connected components of $H-S$ are exactly the connected components of $G-S$.

In particular, all the minimal triangulations of $G$ are efficient [16].

Theorem 2 ([15]). There is an optimal branch decomposition $(T, \tau)$ of $G$ such that the chordal graph $H(T, \tau)$ is an efficient triangulation of $G$. Moreover, each minimal separator of $H$ is the label of some branch of $T$.

Definition 3. A set of vertices $B \subseteq V$ of $G$ is called a block if, for each connected component $C_{i}$ of $G-B$,

- its neighborhood $S_{i}=N\left(C_{i}\right)$ is a minimal separator;

- $B \backslash S_{i}$ is non empty and contained in a connected component of $G-S_{i}$.

We say that the minimal separators $S_{i}$ border the block $B$ and we denote by $\mathcal{S}(B)$ the set of these separators.

Let $\mathcal{B}_{G}$ denote the set of blocks of $G$. Note that $V$ is a block with $\mathcal{S}(V)=\emptyset$.

We prove that if $H$ is an efficient triangulation of $G$, then any maximal clique $K$ of $H$ is a block of $G$.

Lemma 4 ([4]). Let $H$ be a chordal graph and $\Omega$ be a maximal clique of $H$. Then $\Omega$ is a block of $H$.

Lemma 5. Let $H$ be an efficient triangulation of $G$ and $\Omega$ be any maximal clique of $H$. Then $\Omega$ is a block of $G$. Conversely, for any block $B$ of $G$, there is an efficient triangulation $H(B)$ of $G$ such that $B$ induces a maximal clique in $H$.

Proof. If $H$ is an efficient triangulation of $G$, by Lemma 4 every maximal clique $\Omega$ is a block of $H$. By definition of efficient triangulations, a block of $H$ is also a block of $G$.

Conversely, if $B$ is a block of $G$, let $C_{1}, \ldots, C_{p}$ be the connected components of $G-B$ and let $S_{i}=N\left(C_{i}\right)$, for all $1 \leq i \leq p$. Let $H(B)$ be the graph obtain from $G$ by completing $B$ and each set $S_{i} \cup C_{i}$ into a clique. The minimal separators of $H(B)$ are exactly $S_{1}, \ldots, S_{p}$. Moreover, for each $S_{i}$, the connected components of $H-S_{i}$ are exactly the components of $G-S_{i}$.

Note that the treewidth of a graph can be expressed by the following equation:

$$
\operatorname{tw}(G)=\min _{H \text { triangulation of } G} \max \{|\Omega|-1 \mid \Omega \text { maximal clique of } H\} .
$$

The minimum can be taken over all minimal triangulations $H$ of $G$. A similar formula can be obtained for branchwidth.

Definition 6 (block-branchwidth). Let $B$ be a block of $G$ and $K(B)$ be the complete graph with vertex set $B$. A branch decomposition $\left(T_{B}, \tau_{B}\right)$ of $K(B)$ respects the block $B$ if, for each minimal separator $S \in \mathcal{S}(B)$, there is a branch $e$ of the decomposition such that $S \subseteq \operatorname{lab}(e)$. The block branchwidth $\mathrm{bbw}(B)$ of $B$ is the minimum width over all the branch decompositions of $K(B)$ respecting $B$. 
Equivalently, $\operatorname{bbw}(B)$ is the branchwidth of the hypergraph obtained from the complete graph with vertex set $B$ by adding a hyperedge $S$ for each minimal separator $S$ bordering $B$. The blockbranchwidth allows us to express the branchwidth of $G$ by a formula similar to Equation 1 (see Propositions 4.18 and 6.7 in [15]). The proof of the theorem is given in the Appendix.

\section{Theorem 7 ([15]).}

$$
\operatorname{bw}(G)=\min _{H \text { efficient triangulation of } G} \max \{\operatorname{bbw}(\Omega) \mid \Omega \text { maximal clique of } H\} .
$$

A potential maximal clique of a graph $G$ is a set of vertices $\Omega$ such that there is a minimal triangulation $H$ of $G$ in which $\Omega$ introduces a maximal clique [4]. Using the Equation 1, Bouchitté and Todinca show that, given a graph and all its potential maximal cliques, the treewidth of the graph can be computed in polynomial time. The result is refined in [9], where the authors show the following:

Theorem 8. There is an algorithm that, given a graph $G$ and the set $\Pi_{G}$ of its potential maximal cliques, computes the treewidth of $G$ in $\mathcal{O}\left(n m\left|\Pi_{G}\right|\right)$ time.

According to Lemma 5, a vertex subset $\Omega$ of $G$ can be a maximal clique of an efficient triangulation $H$ of $G$ if and only if $\Omega$ is a block of $G$. Hence, in our case the blocks play the same role as the potential maximal cliques in Theorem 8 .

Using Equation 2 instead of Equation 1 and blocks instead of potential maximal cliques, the algorithm cited in Theorem can be directly transformed into an algorithm taking $G$, the set $\mathcal{B}_{G}$ of all its blocks and the block-branchwidth of each block $B$, and computing the branchwidth of $G$ in $\mathcal{O}\left(n m\left|\mathcal{B}_{G}\right|\right)$ time. In the rest of this section we give, without proofs, the new algorithm and the main tools for obtaining it.

Given a minimal separator $S$ of $G$ and a connected component $C$ of $G-S$, let $R(S, C)$ denote the hypergraph obtained from $G[S \cup C]$ by adding the hyperedge $S$.

Lemma 9 (Similar to Corollary 4.5 in [4]). For any graph $G$,

$$
\mathrm{bw}(G)=\min \left(\lceil 2 n / 3\rceil, \min _{S \in \Delta_{G}} \max _{C \in \mathcal{C}(S)} \operatorname{bw}(R(S, C))\right)
$$

Moreover, the minimum can be taken over the inclusion-minimal separators of $G$.

The case when $\operatorname{bw}(G)=\lceil 2 n / 3\rceil$ corresponds to the fact that, for an optimal decomposition $(T, \tau)$ of $G$, the efficient triangulation $H(T, \tau)$ is the complete graph.

Lemma 10 (Similar to Corollary 4.8 in [4]). Let $S$ be a minimal separator of $G$ and $C$ be a component of $G-S$ such that $S=N(C)$. Then

$$
\mathrm{bw}(R(S, C))=\min _{\text {blocks } \Omega \text { s.t. } S \subset \Omega \subseteq S \cup C} \max \left(\mathrm{bbw}(\Omega), \operatorname{bw}\left(R\left(S_{i}, C_{i}\right)\right)\right)
$$

where $C_{i}$ are the components of $G-\Omega$ contained in $C$ and $S_{i}=N\left(C_{i}\right)$.

The algorithm for computing the branchwidth of $G$ is a straightforward translation of Lemmas 9 and 10, and very similar to the one of [9]. 


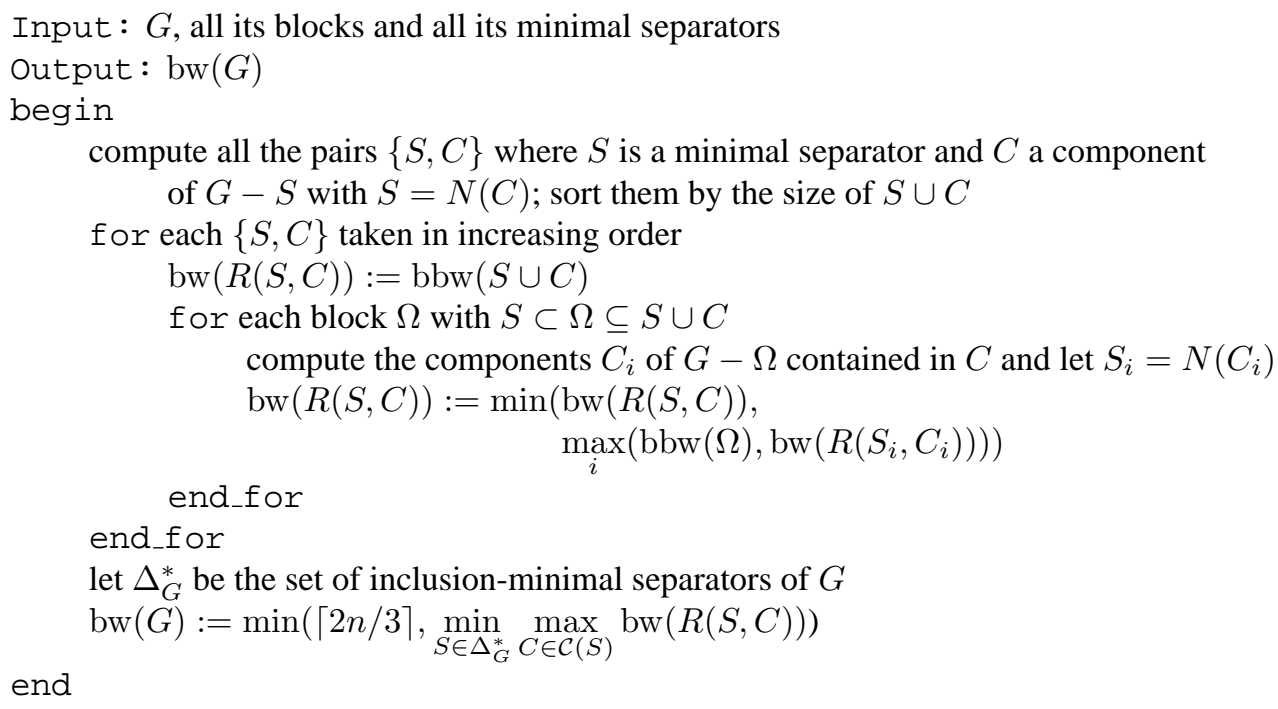

Theorem 11. Given a graph $G$ and the list $\mathcal{B}_{G}$ of all its blocks together with their block-branchwidth, the branchwidth of $G$ can be computed in $\mathcal{O}\left(n m\left|\mathcal{B}_{G}\right|\right)$ time.

Proof. The proof is very similar to the proof for treewidth and potential maximal cliques in [9] and we omit it here.

\section{Computing the block-branchwidth}

The main result of this section is that the block-branchwidth of a block $B$ of $G$ can be computed in $\mathcal{O}^{*}\left(\sqrt{3}^{n}\right)$ time. Computing the block-branchwidth is NP-hard, as it can be deduced directly from [14].

Let $n(B)$ denote the number of vertices of the block $B$ of $G$ and let $s(B)$ be the number of minimal separators bordering $B$. Note that $s(B)$ is at most the number of components of $G-B$, in particular $n(B)+s(B) \leq n$.

Lemma 12. $\operatorname{bbw}(B) \leq p$ if and only if there is a partition of $B$ into four parts $A_{1}, A_{2}, A_{3}, D$ such that

1. $\left|B \backslash A_{i}\right| \leq p$, for all $i \in\{1,2,3\}$;

2. for each minimal separator $S \in \mathcal{S}(B)$, $S$ is contained in $B \backslash A_{i}$ for some $i \in\{1,2,3\}$.

Proof. Suppose that $\operatorname{bbw}(B) \leq p$ and let $(T, \tau)$ be an optimal branch decomposition of $B$ respecting the block. Recall that this branch decomposition corresponds to the complete graph $K(B)$ with vertex set $B$. For each $x \in B$ let $T_{x}$ be the minimal sub-tree of $T$ spanning all the leaves of $T$ labeled with an edge incident to $x$. Let $u$ represent $B$. Clearly $u$ is a ternary node. Let $e_{1}, e_{2}, e_{3}$ be the branches of $T$ incident to $u$. Let $T(i)$ be the sub-tree of $T$ rooted in $u$, containing the branch $e_{i}$, for $i \in\{1,2,3\}$. Let $B_{i}=\{z \in B \mid z$ is incident to some edge of $K(B)$ mapped on a leaf of $T(i)\}$. Fix $D=B_{1} \cap B_{2} \cap B_{3}$, and $A_{i}=B_{j} \cap B_{k} \backslash D$ for all triples $(i, j, k)$ with $i, j, k \in\{1,2,3\}$ and 
distinct. Observe that $D, A_{1}, A_{2}, A_{3}$ form a partition of $B$. The three sets are pairwise disjoint by construction. Since for all $x \in B, u \in T_{x}$, we have that $x \in B_{i} \cap B_{j}$ for distinct $i, j \in\{1,2,3\}$, so $x$ is in one of the four sets $A_{1}, A_{2}, A_{3}$ or $D$. It remains to show that the partition satisfies the conditions of the theorem. Consider a separator $S \in \mathcal{S}(B)$ and a branch $e$ in the decomposition with $S \subseteq$ lab(e). Suppose w.l.o.g. that $e \in T(i)$. Consequently lab $(e) \subseteq B_{i}$, and since $B_{i}=B \backslash A_{i}$ we have the second condition of the theorem. For proving the first condition, since $A_{1}, A_{2}, A_{3}, D$ is a partition of $B$, note that $\operatorname{lab}\left(e_{i}\right)=A_{j} \cup A_{k} \cup D=B \backslash A_{i}$. Therefore $\left|B \backslash A_{i}\right| \leq p$, for all $i \in\{1,2,3\}$.

Conversely, suppose that such a partition exists and let us construct a branch decomposition of $K(B)$ respecting the block $B$, of width at most $p$. Let $B_{i}=B \backslash A_{i}$, for each $i \in\{1,2,3\}$. For each $i$, construct an arbitrary branch decomposition $\left(T_{i}, \tau_{i}\right)$ of the complete graph with vertex set $B_{i}$. Let $T$ be the tree obtained as follows : for each $T_{i}$, add a new node $v_{i}$ on some branch of $T_{i}$, then glue the three trees by adding a new node $u$, adjacent to $v_{1}, v_{2}, v_{3}$. The tree $T$ is a ternary tree and each edge of $K(B)$ is mapped on at least one leaf of $T$, so we obtained a relaxed tree decomposition $(T, \tau)$ of $K(B)$. Let $e_{i}$ be the branch $\left\{u, v_{i}\right\}$. Note that lab $\left(e_{i}\right)=B_{i} \cap\left(B_{j} \cup B_{k}\right)$, where $\{i, j, k\}=\{1,2,3\}$. Hence lab $\left(e_{i}\right)=B_{i}$. Consequently, the relaxed branch decomposition respects the block $B$. Clearly for each branch $e$ of $T, \operatorname{lab}(e)$ is contained in some $B_{i}$, so $|\operatorname{lab}(e)| \leq$ $p$ and the conclusion follows.

Theorem 13. The block-branchwidth of any block $B$ can be computed in $\mathcal{O}^{*}\left(3^{s(B)}\right)$ time.

Proof. Let $B$ be a block of $G$. Suppose that $\operatorname{bbw}(B) \leq p$. By Lemma 12, there exists a partition of $B$ in $A_{1}, A_{2}, A_{3}$ and $D$ such that $\left|B \backslash A_{i}\right| \leq p$ and every $S \in \mathcal{S}(B)$ is a subset of $B \backslash A_{i}$. Denote by $a_{1}, a_{2}, a_{3}$ and $d$ the sizes of $A_{1}, A_{2}, A_{3}$ and $D$. We can partition $\mathcal{S}(B)$ in three subsets $\mathcal{S}_{i}$ such that every $S \in \mathcal{S}_{i}$ is included in $B \backslash A_{i}$. Let $S_{i}$ be the union of all the minimal separators of $\mathcal{S}_{i}$. The numbers $a_{1}, a_{2}, a_{3}$ and $d$ satisfy the following inequalities:

1. $a_{i} \geq 0, d \geq 0, a_{1}+a_{2}+a_{3}+d=|B|$;

2. $\left|S_{1} \cap S_{2} \cap S_{3}\right| \leq d,\left|\left(S_{1} \cap S_{2}\right) \backslash S_{3}\right| \leq a_{3},\left|\left(S_{2} \cap S_{3}\right) \backslash S_{1}\right| \leq a_{1},\left|\left(S_{3} \cap S_{1}\right) \backslash S_{2}\right| \leq a_{2}$;

3. $a_{1}+a_{2}+d \leq p, a_{2}+a_{3}+d \leq p, a_{3}+a_{1}+d \leq p$.

The first inequalies express the fact that $A_{1}, A_{2}, A_{3}$ and $D$ is a partition of $B$, the second express the fact that $S_{i}$ is a subset of $B \backslash A_{i}$ and the last ones express the fact that $\operatorname{bbw}(B) \leq p$.

Conversely, suppose there is a partition of $\mathcal{S}(B)$ in $\mathcal{S}_{1}, \mathcal{S}_{2}$ and $\mathcal{S}_{3}$ and four integers $a_{1}, a_{2}, a_{3}, d$ satisfying the system above. Then there exist a partition of $B$ into four sets $A_{1}, A_{2}, A_{3}, D$, of cardinalities $a_{1}, a_{2}, a_{3}, d$ and such that $D$ intersects $S_{1} \cup S_{2} \cup S_{3}$ exactly in $S_{1} \cap S_{2} \cap S_{3}$, and each $A_{i}$ intersects $S_{1} \cup S_{2} \cup S_{3}$ exactly in $\left(S_{j} \cap S_{k}\right) \backslash S_{i}$, where $\{i, j, k\}=\{1,2,3\}$. Moreover $\left|B \backslash A_{i}\right| \leq p$ by the third series of inequalities, so by Lemma 12 we have $\operatorname{bbw}(B) \leq p$.

Hence, there an efficient branch decomposition of $K(B)$ respecting $B$ of branchwidth at most $p$ if and only if there is a partition partition $\mathcal{S}_{1}, \mathcal{S}_{2}, \mathcal{S}_{3}$ of $\mathcal{S}(B)$ and four numbers $a_{1}, a_{2}, a_{3}$ and $d$ satisfying the system. To decide whether $\operatorname{bbw}(B) \leq p$ or not, we only have to try all the partitions of $\mathcal{S}(B)$ in $\mathcal{S}_{1}, \mathcal{S}_{2}$ and $\mathcal{S}_{3}$ and check all the $n^{4}$ possible values for the $a_{i}$ 's and $d$. This can be done in $\mathcal{O}^{*}\left(3^{|\mathcal{S}(B)|}\right)=\mathcal{O}^{*}\left(3^{s(B)}\right)$ time as claimed. 
Theorem 14. The block-branchwidth of any block B can be computed in $\mathcal{O}^{*}\left(3^{n(B)}\right)$ time.

Proof. We show that for any number $p$, the existence of a partition like in Lemma 12 can be tested in $\mathcal{O}^{*}\left(3^{n(B)}\right)$.

For this purpose, instead of partitioning $B$ into four parts, we try all the partitions of $B$ into three parts $A_{1}, X, D$, where $X$ corresponds to $A_{2} \cup A_{3}$. If $\left|B \backslash A_{1}\right| \leq p$, we check in polynomial time if $X$ can be partitioned into $A_{2}$ and $A_{3}$ as required. Since there are at most $3^{n(B)}$ threepartitions of $B$, it only remains to solve this last point.

We say that two vertices $x, y \in X$ are equivalent if there exist $z \in A_{1}$ and a minimal separator $S$ bordering $B$ such that $x, y, z \in S$. In particular, $x \sim y$ implies that $x$ and $y$ must be both in $A_{2}$ or both in $A_{3}$. Let $X_{1}, \ldots, X_{q}$ be the equivalence classes of $X$. Then $X$ can be partitioned into $A_{2}$ and $A_{3}$ as required if and only if $\left\{\left|X_{1}\right|, \ldots,\left|X_{q}\right|\right\}$ can be partitioned into two parts of sum at most $p-\left|A_{1}\right|-|D|$ vertices. Consider now the EXACT SUBSET-SUM problem, whose instance is a set of positive integers $I=\left\{i_{1}, \ldots, i_{q}\right\}$ and a number $t$, and the problem consists in finding a subset of $I$ whose sum is exactly $t$. Though NP-hard in general, it becomes polynomial when $t$ and the numbers $i_{j}$ are polynomially bounded in $n$ (see e.g. the chapter on approximation algorithms, the subset-sum problem in the book of Cormen, Leiserson, Rivest [6]). By taking $I=\left\{\left|X_{1}\right|, \ldots,\left|X_{q}\right|\right\}$ and trying all possible values of $t$ between 1 and $n^{2}$, we can check in polynomial time if $X$ can be partitioned as required.

Since at least one of $s(B)$ or $n(B)$ is smaller or equal to $n / 2$, we deduce:

Theorem 15. For any block $B$ of $G$, the block-branchwidth of $B$ can be computed in $\mathcal{O}^{*}\left(\sqrt{3}^{n}\right)$ time.

Theorems 11 and 15 imply our main result.

Theorem 16. The branchwidth of graphs can be computed in $\mathcal{O}^{*}\left((2+\sqrt{3})^{n}\right)$ time and $\mathcal{O}^{*}\left(2^{n}\right)$ space.

Proof. The algorithm enumerates every subset $B$ of $V$ and checks if $B$ is a block. Clearly, we can verify if $B$ is a block in polynomial time. If so, we compute the block branchwidth of $B$ using Theorem 15. The number of blocks is at most $2^{n}$ and for each block we need $\mathcal{O}^{*}\left(\sqrt{3}^{n}\right)$ for computing its block branchwidth. Hence the running time of this phase is $\mathcal{O}^{*}\left((2+\sqrt{3})^{n}\right)$, and the space is $\mathcal{O}^{*}\left(2^{n}\right)$.

Eventually, we use Theorem 11 for computing the branchwidth of $G$. The second phase takes $\mathcal{O}^{*}\left(2^{n}\right)$ time and space.

\section{Open problems}

Our algorithm is based on the enumeration of the blocks of a graph (in $\mathcal{O}^{*}\left(2^{n}\right)$ time) and on the computation of the block-branchwidth of a block (in $\mathcal{O}^{*}\left(\sqrt{3}^{n}\right)$ time). It is natural to ask whether one of these steps can be improved.

Computing the block-branchwidth is the same problem as computing the branchwidth of a complete hypergraph with $n^{\prime}$ vertices and $s^{\prime}$ hyper-edges of cardinality at least three. Can we obtain an algorithm faster than our $O\left(\max \left(3^{n^{\prime}}, 3^{s^{\prime}}\right)\right)$-time algorithm? 
Note that there exist graphs with $n$ vertices having $2^{n} / n^{O(1)}$ blocks. Indeed, consider the disjoint union of a clique $K$ and an independent set $I$, both having $n / 2$ vertices, and add a perfect matching between $K$ and $I$. We obtain a graph $G_{n}$ such that for any $I^{\prime} \subseteq I, G_{n}-I^{\prime}$ is a block. Thus $G_{n}$ has at least $\left(\begin{array}{c}n \\ n / 2\end{array}\right) \geq 2^{n} / n$ blocks. The interesting question here is if we can define a new class of triangulations, smaller than the efficient triangulations but also containing $H(T, \tau)$ for some optimal branch decompositions of the graph.

\section{References}

[1] R. Beigel and D. Eppstein. 3-coloring in time $O\left(1.3446^{n}\right)$ : a no-MIS algorithm. Proceedings of the 36th IEEE Symposium on Foundations of Computer Science (FOCS 1995), pp. 444452.

[2] R. Beigel and D. Eppstein. 3-coloring in time $O\left(1.3289^{n}\right)$. Journal of Algorithms, 54:444453, 2005.

[3] H. L. Bodlaender, A partial $k$-arboretum of graphs with bounded treewidth, Theoret. Comput. Sci., 209:1-45, 1998.

[4] V. Bouchitté and I. Todinca. Treewidth and minimum fill-in: grouping the minimal separators. SIAM J. on Computing, 31(1):212 - 232, 2001.

[5] J. M. Byskov. Enumerating maximal independent sets with applications to graph colouring. Operations Research Letters, 32:547-556, 2004.

[6] T. Cormen, C. Leiserson, and R. Rivest. Introduction to algorithms. The MIT press, 1990.

[7] E. Dantsin, A. Goerdt, E. A. Hirsch, R. Kannan, J. Kleinberg, C. Papadimitriou, P. Raghavan, and U. Schöning. A deterministic $(2-2 /(k+1))^{n}$ algorithm for k-SAT based on local search. Theoretical Computer Science, 289(1):69-83, 2002.

[8] D. Eppstein. Improved algorithms for 3-coloring, 3-edge-coloring, and constraint satisfaction. Proceedings of the 12th ACM-SIAM Symposium on Discrete Algorithms (SODA 2001), pp. 329-337.

[9] F. Fomin, D. Kratsch, and I. Todinca. Exact (exponential) algorithms for treewidth and minimum fill-in. In Proceedings 31st International Colloquium on Automatas, Languages and Programming (ICALP'04), volume 3142 of Lecture Notes in Computer Science, pages 568-580. Springer, 2004.

[10] F. Gavril. The intersection graphs of a path in a tree are exactly the chordal graphs. Journal of Combinatorial Theory, 16:47-56, 1974.

[11] M. C. Golumbic. Algorithmic Graph Theory and Perfect Graphs. Academic Press, New York, 1980.

[12] K. Iwama and S. Tamaki. Improved upper bounds for 3-SAT. Proceedings of the 15th ACMSIAM Symposium on Discrete Algorithms (SODA 2004), p.328. 
[13] T. Jian. An $\mathrm{O}\left(2^{0.304 n}\right)$ algorithm for solving maximum independent set problem. IEEE Transactions on Computers, 35(9):847-851, 1986.

[14] T. Kloks, J. Kratochvíl, and H. Müller. New branchwidth territories. In Proceedings 16th Annual Symposium on Theoretical Aspects of Computer Science (STACS '99), volume 1563 of Lecture Notes in Computer Science, pages 173-183. Springer, 1999.

[15] F. Mazoit. Décompositions algorithmiques des graphes. $\mathrm{PhD}$ thesis, École normale supérieure de Lyon, 2004. In French.

[16] A. Parra and P. Scheffler. Characterizations and algorithmic applications of chordal graph embeddings. Discrete Appl. Math., 79(1-3):171-188, 1997.

[17] R. Paturi, P. Pudlak, M. E. Saks, and F. Zane. An improved exponential-time algorithm for kSAT. Proceedings of the 39th IEEE Symposium on Foundations of Computer Science (FOCS 1998), pp. 628-637.

[18] N. Robertson and P. Seymour. Graph minors X. Obstructions to tree decompositions. Journal of Combinatorial Theory Series B, 52:153-190, 1991.

[19] J. M. Robson. Algorithms for maximum independent sets. Journal of Algorithms, 7(3):425440, 1986.

[20] U. Schoning. A Probabilistic Algorithm for k-SAT and Constraint Satisfaction Problems. Proceedings of the 40th IEEE Symposium on Foundations of Computer Science (FOCS 1999), pp. 410-414.

[21] P. D. Seymour and R. Thomas, Call routing and the ratcatcher, Combinatorica, 14:217-241, 1994.

[22] R. Tarjan and A. Trojanowski. Finding a maximum independent set. SIAM Journal on Computing, 6(3):537-546, 1977.

[23] R. Williams. A new algorithm for optimal constraint satisfaction and its implications. Proceedings of the 31st International Colloquium on Automata, Languages and Programming (ICALP 2004), Springer LNCS vol. 3142, 2004, pp. 1227-1237.

[24] G. J. Woeginger. Exact algorithms for NP-hard problems: A survey. Combinatorial Optimization - Eureka, You Shrink, Springer LNCS vol. 2570, 2003, pp. 185-207. 


\section{A Appendix}

Theorem 7 ([15]).

$$
\operatorname{bw}(G)=\min _{H \text { efficient triangulation of } G} \max \{\operatorname{bbw}(\Omega) \mid \Omega \text { maximal clique of } H\} .
$$

Proof. Let $(T, \tau)$ be an optimal branch decomposition of $G$ such that $H=H(T, \tau)$ is an efficient triangulation of $G$. Such a decomposition exists by Theorem 2. First, let us construct a branch decomposition $\left(T^{\prime}, \tau^{\prime}\right)$ of $H$ having the same width as $(T, \tau)$. For each edge $\{x, y\}$ of $E(H)-$ $E(G)$, the sub-trees $T_{x}$ and $T_{y}$ share a branch $e$. We divide the branch $e$ by a node $v$, add a leaf $w$ adjacent to $v$ and map the edge $\{x, y\}$ on $w$. Clearly this will not increase the width of the decomposition. Consider any maximal clique $\Omega$ of $G$. By Lemma $5, \Omega$ is a block of $G$ and by Theorem 2 each minimal separator bordering $\Omega$ is contained in the label of some branch $e_{S}$ of $T^{\prime}$. For each $S$ let $\left(T_{S}, \tau_{S}\right)$ be a arbitrary branch decomposition of the clique $K(S)$. We glue this decomposition to $T^{\prime}$ on the branch $e_{S}$. That is, we add a node on $e_{S}$ and a node on some branch of $T_{S}$ and make them adjacent. We call this new edge $e_{S}^{\prime}$, in particular its label is exactly $S$. By this process we obtain a relaxed branch decomposition $\left(T^{\prime \prime}, \tau^{\prime \prime}\right)$ of $H$ of same width as $\left(T^{\prime}, \tau^{\prime}\right)$. By removing from $T^{\prime \prime}$ all the leaves that do not correspond to edges in the clique $\Omega$, we obtained a relaxed clique decomposition of the complete graph $K(\Omega)$. For each minimal separator $S$ bordering $\Omega$, note that $S$ is contained in the label of the edge $e_{S}^{\prime}$, so the new decomposition respects $\Omega$. Hence bbw $(\Omega) \leq \operatorname{bw}(G)$ for each maximal clique $\Omega$ of $H$.

Conversely, let $H$ be any efficient triangulation of $G$, let us show that $\operatorname{bw}(G) \leq \max \{\operatorname{bbw}(\Omega) \mid$ $\Omega$ maximal clique of $H$ \}. For each maximal clique $\Omega$ of $G$, let $\left(T_{\Omega}, \tau_{\Omega}\right)$ be an optimal branch decompoition of $K(\Omega)$, respecting the block $\Omega$. We connect these decompositions into a relaxed branch decomposition of $H$. For this purpose we use a clique tree associated to the chordal graph graph $H$ (see e.g. [11]). A clique tree is given by a tree $T=\left(V_{T}, E_{T}\right)$ and a one-to-one correspondence between the nodes of $T$ and the maximal cliques of $H$ such that, for each $\Omega, \Omega^{\prime}$ maximal cliques of $H$, their intersection is contained in all the cliques associated to nodes on the unique path from $u_{\Omega}$ to $u_{\Omega^{\prime}}$ of $T$ ( $u_{\Omega}$ and $u_{\Omega^{\prime}}$ denote the nodes associated to $\Omega$ and $\Omega^{\prime}$ respectively). Moreover, for each branch $e=\left\{u_{\Omega}, u_{\Omega^{\prime}}\right\}$ of $T, S=\Omega \cap \Omega^{\prime}$ is a minimal separator bordering $\Omega$ and $\Omega^{\prime}$ [11]. Let $e_{S}$ (resp. $e_{S}^{\prime}$ ) be a branch of $T_{\Omega}$ (resp. $T_{\Omega^{\prime}}$ ) whose label contains $S$. We connect $T_{\Omega}$ and $T_{\Omega^{\prime}}$ by adding a new branch between the middle of $e_{S}$ and $e_{S}^{\prime}$, for all branches $\left\{u_{\Omega}, u_{\Omega^{\prime}}\right\}$ of $T$. Hence we obtain a relaxed branch decomposition of $H$. By the properties of the clique tree, the label of each newly created edge connecting $T_{\Omega}$ and $T_{\Omega^{\prime}}$ is exactly $S=\Omega \cap \Omega^{\prime}$. Consequently, the labels of the branches contained in some $T_{\Omega}$ do not change. Hence $\operatorname{bw}(H) \leq \max \{\operatorname{bbw}(\Omega) \mid \Omega$ maximal clique of $H\}$. $G$ being a sub-graph of $H$, we have $\mathrm{bw}(G) \leq \mathrm{bw}(H)$ and the conclusion follows. 